\title{
Rapid prototyping for the extension of the accessibility to cultural heritage for blind people
}

\author{
A. Scianna ${ }^{1}$, G. Di Filippo² \\ ${ }^{1}$ ICAR-CNR (High Performance Computing and Networking Institute - National Research Council of Italy) at GISLab c/o D’Arch, \\ Polytechnic School of University of Palermo, Viale delle Scienze, Edificio 8, 90128 Palermo, Italy \\ andrea.scianna@cnr.it \\ 2 Dept. of Engineering, Polytechnic School of University of Palermo, Viale delle Scienze, Edificio 9, 90128 Palermo, Italy \\ gabriella.df@hotmail.it
}

\section{Commission II, WG II/8}

KEY WORDS: 3D Printing, Rapid Prototyping, Additive Manufacturing, Cultural Heritage, Accessibility, 3D.

\section{ABSTRACT:}

The extension of accessibility of $\mathrm{CH}$ to disabled people is a current topic of great importance. Today, there exist a lot of ongoing projects aimed at the extension of accessibility using the most recent and low cost technologies to improve accessibility to $\mathrm{CH}$. One of the technologies, today available that can help to improve accessibility to $\mathrm{CH}$, is certainly rapid prototyping. The main goal of the research here illustrated is the application of rapid prototyping for the extension of accessibility of Cultural Heritage $(\mathrm{CH})$ to blind and visually impaired people. This document reports an experiment that compared the effectiveness of different 3D models for the introduction of blind and visually impaired people to the tactile use of models of monuments. In addition, this study focuses on defining standards and guidelines regarding features of 3D models, e.g. print resolution, in order to obtain greater legibility of models at different scales by the blind or visually impaired people. This paper shows a part of a wider Interreg EU research project, named I-ACCESS, aimed to study accessibility problems and solutions for $\mathrm{CH}$. The experiments conducted with the sample of disabled people, allowed to obtain results on the most suitable printing parameters to be used.

\section{INTRODUCTION}

In recent years, great attention has been taken to the problem of the removal of architectural barriers. This was one of the most significant interventions of the Italian Ministry of Cultural Heritage and Activities (MiBACT). A ministerial Commission, established in 2018, published guidelines for overcoming architectural barriers in places of cultural interest (P.E.B.A.). Physical, sensorial and cultural accessibility are to be considered essential to make the places of culture fully accessible to all visitors (MiBACT). Besides, accessibility to $\mathrm{CH}$ is sustained by Council of Europe that adopted a new "Strategy on the Rights of Persons with disabilities 2017-2023”.

The elimination of barriers for physical disabilities that involves some architectural interventions (removal of stairs, obstacles) could be easily achieved, while it is much more difficult to reduce the barriers that are mainly perceptive as in the case of people with visual defects.

The removal of architectural barriers, both physical and cognitive, positively improves the impact of accessibility on tourism, with significant effects on the economy (contributes to further development of tourism, improves public services, creates new opportunities for employment for all), social (promotes social cohesion, improves the quality of life, reduces the feeling of social exclusion), cultural (offers a knowledge of culture and heritage, offers the feeling of being part of the collective cultural capital, increases cultural capital individual) and environmental (correct access for all to spaces of high cultural importance, visual aesthetic improvement of cultural spaces, increase of aesthetic awareness of natural environments) (Remondino, 2011). About the improvement of physical accessibility, a great contribution can be granted, by the recent evolution of rapid prototyping that can be seen, today, as low cost technology. Indeed, the recent evolution of 3D terrestrial laser scanners (TLS) and related software, photogrammetric restitution techniques based on computer vision algorithms and low-cost 3D printers has brought great progress in rapid prototyping applications.

In education, different approaches have been designed for the production of tactile material for people with visual impairments through the generation of 3D printing content (Neumüller et al., 2014).

Obviously, the main aspect is how to reproduce models that are adapt to be explored and understood, during tactile experience, by visually impaired and blind people that have developed their senses in order to cope visual disability. In fact, requirements of models, suitable to tactile exploration by blind/visually impaired people, are different from models that can be visually appreciated by sighted people. Blind or visually impaired people cannot appreciate colours except some specific cases regarding visually impaired people and the resolution of every model to be explored is related to their fingers and brain sensitivity. So models for tactile experience must be must be appropriately designed and realized carrying out also, some tests with blind or visually impaired people.

The work presented in this article studies the methods and behaviours, of blind people, while exploring tactile 3D models and determines their needs, for 3D model construction, in order to grant them a better accessibility to $\mathrm{CH}$. In addition, this study focuses on defining standards and guidelines regarding features of 3D models, e.g. print resolution, in order to obtain greater legibility of models at different scales by the blind or visually impaired people.

Specifically, we address three current research questions: 1 - how blind and visually impaired people explore tactile models; 2 geometric parameters suitable for blind people to recognize architectural parts; 3 - which printing parameters must be used to make easily accessible the architecture of a specific monument. In fact, the tactile exploration of some printed elements may be difficult, due to the size too large (for example entire buildings, sculptures, etc.) or too small (for example decorative elements such as capitals, metopes, triglyphs, etc.). To develop this

\footnotetext{
* Corresponding author
} 
research, sample printed models were subjected to the exploration by people with visual impairments, both blind and visually impaired. In particular, the facades of some historical and artistic interest monuments, comprised in a route, of IACCESS project, in the historical center of the city of Palermo, have been surveyed using laser scanners, and reproduced in polylactic acid (PLA). 3D models have been realized using free and open source software and they were printed at different scales of representation, different resolution and level of detail. So blinded people were observed while performing tactile exploration of 3D models, letting us to be guided by their tactile perceptions and sensations to better understand, also with the help of interviews, what could be the best solution among those proposed by us. On the basis of the experiences carried out, therefore, some useful indications have been fixed to identify a protocol of surveying, modelling and 3D printing specifications for historical-monumental itinerary inside the historical centre like that of Palermo or museums.

\section{BACKGROUND AND RELATED WORK}

\subsection{How blind and visually impaired people perceive object}

The concept of blindness can be described as the total or partial lack of visual perception, which can't be corrected with lenses or glasses (Medline, 2014). This means that the perception of the world by people affected by this disability occurs through the auditory, kinaesthetic and tactile sense (Amiralian, 2009). In the education sector, blind people use the Braille system to write and read and other devices such as tactile images, audio descriptions and automatic readers. Blindness can be of two types: congenital and acquired. Congenital blindness refers to those who are born blind or lost vision until the age of five and has considerable repercussions on the cognitive processing of space; acquired blindness occurs after a person loses his vision after the age of five and is closely related to the experiences lived by the subject before becoming blind. A low vision is defined as a heterogeneous set of serious reductions in visual function that can't be eliminated by means of corrective lenses, surgical interventions or therapies (Amiralian, 1997). In both cases, the use of technological and tactile aids contributes to improving the quality of life of the blind or visually impaired, helping autonomy, independence and inclusion.

A lot of studies exist concerning the nature of mental visual images in individuals who have never seen (congenital blindness) and those who have reduced vision or who have lost it completely as adults. Many researchers have been interested in tactile perception in the blind, studying the sense of touch in people without visual experience or visual images (Heller, 1991). Some blind people have never seen. These persons must base their understanding of space on the senses of touch, kinaesthetic, hearing and smell. We have always wondered if the blind imagine objects as us, if they understand space in the same way we do and if they have mental images. The mental imagination is a critical function of human cognition and is often considered as a "almost perceptive" experience of objects in their physical absence. "Seeing with the eyes of the mind" or "having an image in one's head" are all commonly used expressions to define this type of images. There are numerous questions concerning the nature of mental images in individuals who have never seen or have always had reduced vision (Hollins, 1985). One study compared performances of individuals with congenital blindness and those with delayed blindness; the aim was to understand if the lack of visual experience affects the ability to create spatial representations. This study showed that the differences between people with congenital and late blindness were not significant and that blind individuals rely strongly on mental images, although some processes of imagination may be more difficult in the absence of visual experience (Tinti et al., 2006).

\subsection{Theoretical framework}

In the last few years, the development of 3D geomatics technologies applied to the $\mathrm{CH}$ sector has led to important results and the 3D printing process has acquired increasingly high levels of detail and accuracy (Remondino, 2011). Thanks to the development of advanced survey technologies, mainly terrestrial/aerial laser scanning and photogrammetry, 3D reconstruction has become simpler and digital models today can have a high level of accuracy (Dore and Murphy, 2012).

Laser scanner technology allows a rapid elaboration of digital models of surfaces and complex geometries which would be impossible to survey with traditional topographic instruments. It therefore represents the proper technology for surveying archaeological sites and objects of Cultural Heritage. The process for the realization of the model is already known in the literature (Peloso, 2005). With further advances in 3D printing, today, copies of buildings or parts very similar to the originals can be easily obtained (Balletti et al., 2017). Starting from points clouds, obtained surveying monuments with the most sophisticated laser scanner instruments or photogrammetry surveys, it is possible to create 3D models in different materials (PLA, ABS, polyamide, etc.), useful for exploration by blind or visually impaired people (Neumüller et al., 2014). Currently, there are numerous studies on the possibility of using 3D printing as a teaching tool and on its' potential within a context of people with visual disabilities. Seeing the potential of 3D models, some researchers have developed methods to improve them by integrating models with audio descriptions. They created interactive 3D printed models equipped with sensors such as cameras (Reichinger et al., 2016; Shi et al. 2016 a), capacitive sensors and microphones (Shi, 2015; Shi, 2016b). These interactive models can also perceive the gestures of a blind user and communicate the corresponding audio information to him. Through the creation of 3D models, it is possible to help the blind or visually impaired people to understand complex concepts such as the architectural elements of an ancient facades of buildings, which can't be deduced usually, only from a text or an audio, giving the opportunity to touch the shape of monuments that otherwise would remain unexplored and inaccessible. Each object to be printed has its own characteristics and with respect to these, tools and printing methods must be chosen to express, at best, its physical characteristics: the model of each $\mathrm{CH}$ piece must respond in terms of accuracy and level of detail to the requirements necessary for the various scales of representation. In the case of large surveys, a multi-scale representation can be also requested.

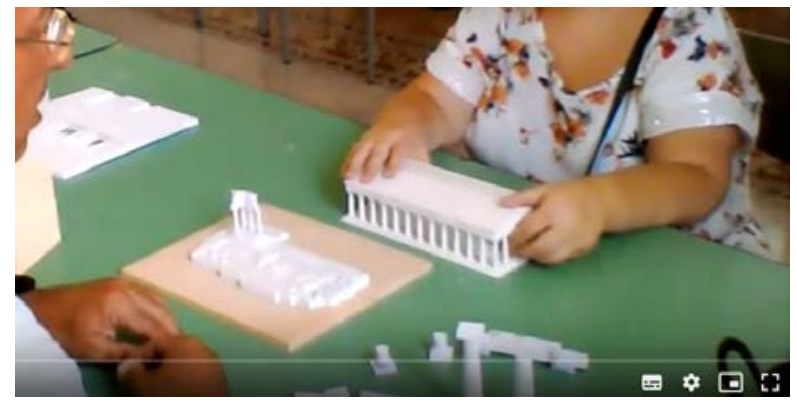

Figure 1. Tactile exploration of 3D models by blind person 


\section{THE CASE STUDY}

In order to carry out this research, some monuments included in a cultural itinerary that winds along the streets of Vucciria in Palermo have been surveyed, restituted and printed in 3D.

These monuments are churches, fountains, but also temples and some detailed building parts that were present also in most recent monumental buildings (e.g. columns or triangular portals).

The models, during also proposition of a questionnaire, were subjected to the exploration by people with visual impairments, both blind and visually impaired (see Figure 1).

The questionnaire has been structured in a way to understand times of blindness evolution, level of culture or interest toward $\mathrm{CH}$, pre-existing knowledge on $\mathrm{CH}$ on every interviewed person. We observed the participants while performing different tasks from the exploration and identification of 3D printed models and their components, without giving a preliminary information on proposed model and after some information on piece already explored.

One of the monumental parts adopted as case study, the facade of the church of San Sebastiano, a church dating back to the 1500s and placed inside the Arab Norman route in Palermo, complex in terms of architectural conservation as it was damaged by the bombing during the second world War was surveyed and printed in scale different scales.
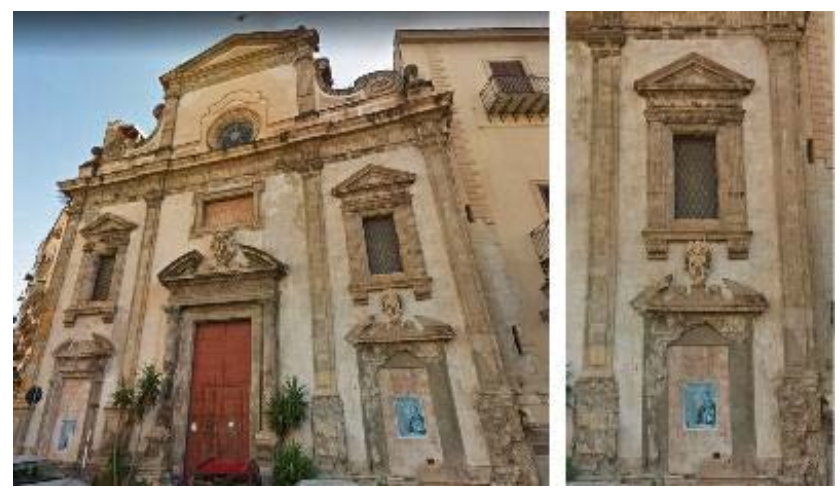

Figure 2. Church of San Sebastiano in Palermo (IT) - facade detail

Acquisition of geometric data of monument implied a lot of difficulties operating in an urban environment where are present a lot of obstructions or limitation due also to reduced space inside which is possible to operate.

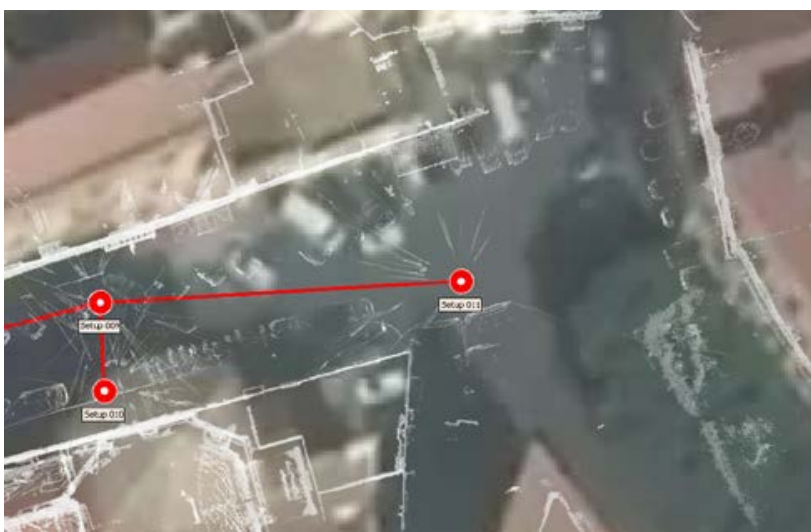

Figure 3. Survey station used for facade reconstruction

In the case of Figure 3, being impossible to adopt a station point in a very high position, in order to obtain a model without holes, scans were performed at different distances so to better scan the hidden parts. Missing part of meshes were added with Blender.
We have therefore created models printed on different scale and resolution, which we then subjected to a sample of people with visual disabilities, letting ourselves be guided by their tactile perceptions to better understand what could be the best solution among those we have proposed. Each object to be printed has its own characteristics and with respect to these it is necessary to choose the tools and printing methods suitable to best express its consistence: the object model must respond in terms of precision and detail to the requirements necessary for the various representations. In the case of large surveys, a multiscale representation scale has been adopted. The tools used are all open source software that allow the modification of point clouds by laser scanner, in. las or. e57 format which are then transformed into meshes and then into 3D solids before slicing.

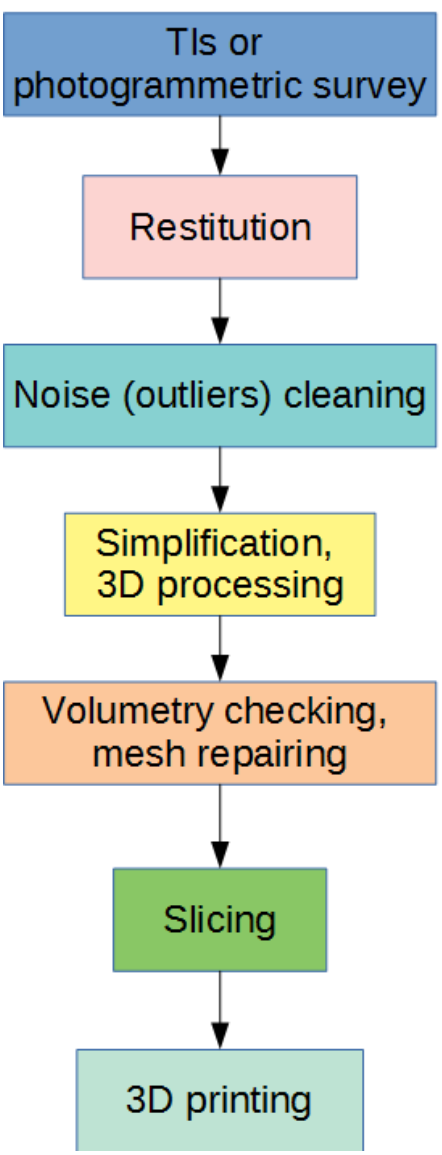

Leica Cyclone

Register 360

Autodesk Recap

Leica Cyclone

Register 360

Meshlab, Cloud

Compare?

Blender ?

Autodesk

Meshmixer

Cura 4.0

Figure 4. Rapid Prototyping workflow

\section{METHODOLOGY}

The workflow of rapid prototyping is more complex in respect to a simple 3D graphics model production as illustrated in Figure 4. The field survey was carried out with a Terrestrial laser scanner Leica RTC360 3D Laser Scanner which made it possible to obtain a point cloud at a resolution of $6-10 \mathrm{~mm}$ with cloud errors under $2 \mathrm{~mm}$ (see Figure 5 and 6). After the field survey (laser scanner and photogrammetric survey), each scan was cleaned of the anomalous values produced by the noise until an optimal point cloud was reached.

Subsequently, the triangular mesh was created and the closing of the holes made it possible to obtain the surface mesh model. To do this, Cloud Compare (a free and open source 3D point and mesh processing software) was used. 


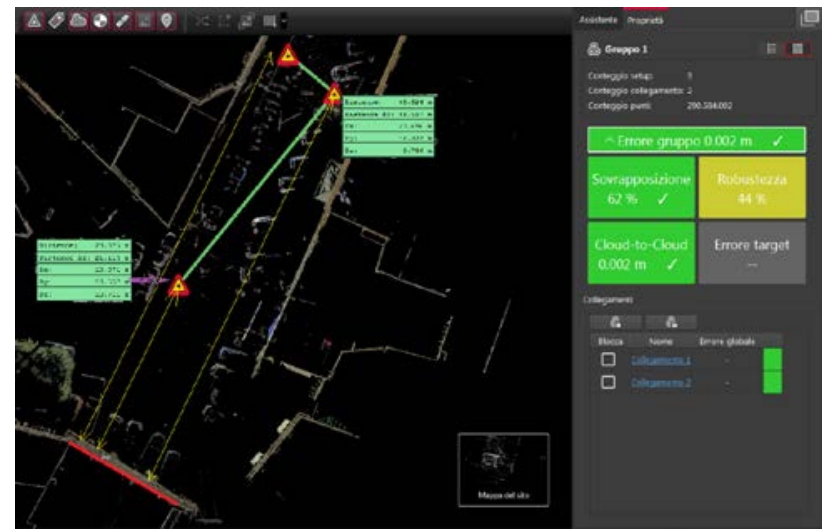

Figure 5. TLS survey processing information

Finally, the transformation of the 3D surface model into a solid, made through the use of the Mesh Mixer software, the solid was cut and printed in PLA. Due to the size of the printer plate $(22 \times 28$ $\mathrm{cm}$ ), the model was divided into different parts and then pasted together. Several models have been printed in 1:50, 1:75, 1:100 scale and with different resolutions.

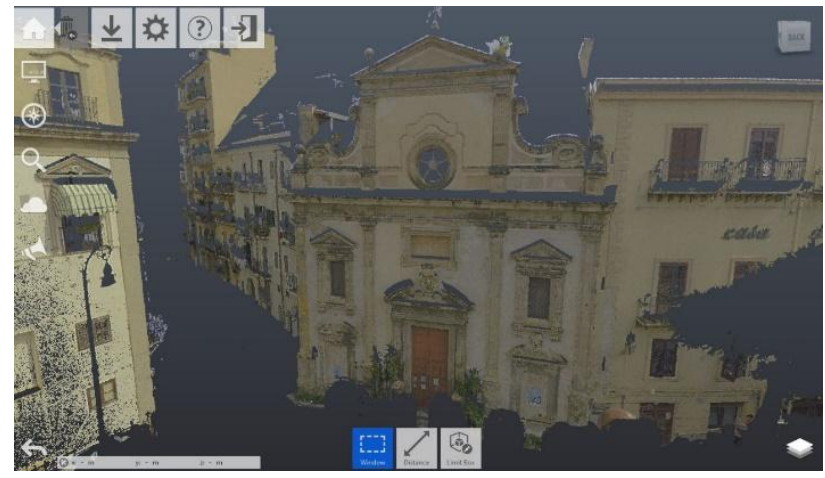

Figure 6. Point cloud of Church of San Sebastiano

A particular model was printed accentuating the volumes and extruding them more. The CURA software was used for 3D printing, with a layer height of 0,1 mm (see Figure 7).
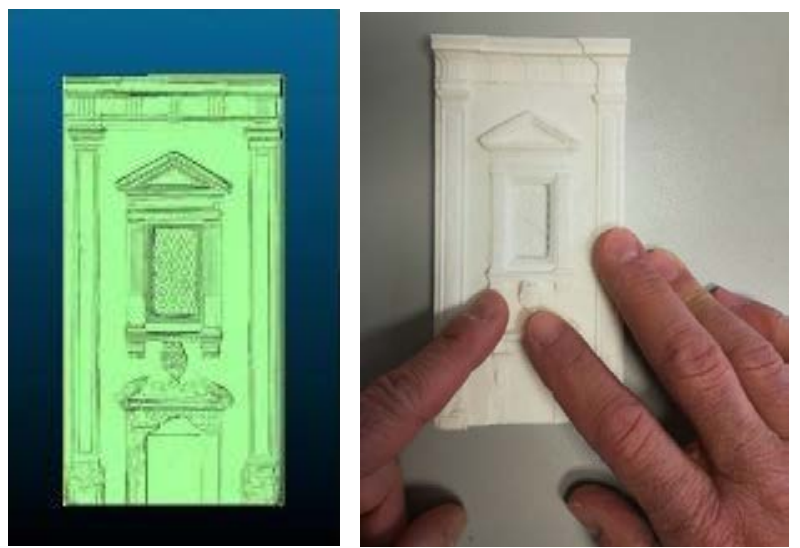

Figure 7. 3D model and 3D printed model

As said before tactile tests were carried out in two steps. The first one, in which blind or visually impaired people were not informed about the object to explore (see Figure 8 and 9). The second one after people were informed about the object to explore with the same information that could be transmitted in place with Braille short texts or with the help of mobile phone.
Having obtaining the 3D printed models, an interview was conducted on a sample of blind and partially sighted people asking them if they could recognize the models represented. According to a study already conducted (Hatwell et al. 2003), it was found that tactile exploration includes two phases, an overview and a detailed exploration; the participants would stop exploring in the first phase if they had not received further instructions. For this reason, participants were asked to explore the models as a whole and to examine the smaller components later.

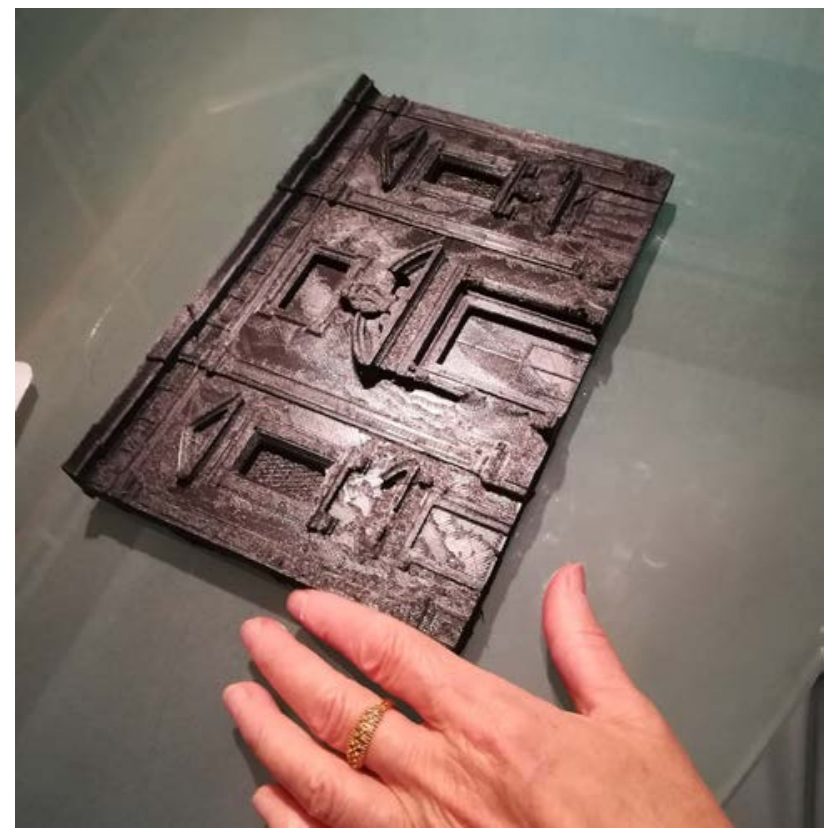

Figure 8. 3D printed model - experiment with a blind person

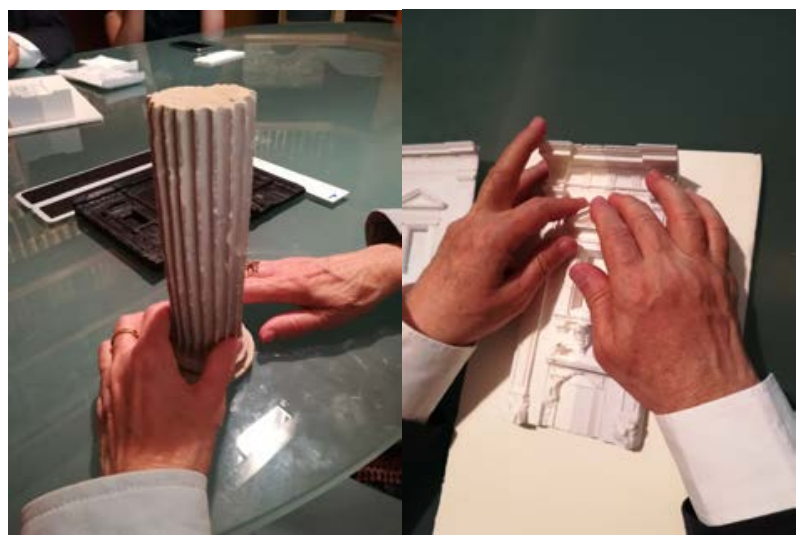

Figure 9. 3D printed model - experiment with a blind person

Furthermore, it was asked to understand if the $1: 50,1: 75$ or $1: 100$ scale was valid for that type of model. Two questionnaires were then submitted, a first survey with no instructions (questionnaire 1 ) and a second giving explanations on the 3D model (questionnaire 2). In this survey phase, a sample of $10 / 15$ people affected by different types of visual impairment was involved.

\section{RECOGNITION SURVEY AND RESULTS}

Based on the experiences, some useful indications were the identification of the 3D modelling protocol and the printing specifications. Initially disabled people suggested to make available in front of each monument a 3D printed map of the plan 
of each building in order to allow them to have a clear idea how to move around.

First, the possibility of accentuating the impression was excluded, as this was less readable than a model printed with normal extrusion. The expanded model in the third direction is too angular and the overhangs are not understandable. The blind from adolescence retain memory of the size of some monuments (temples, churches) and colours. Whilst blind people from birth have no idea of colour combinations and they have no idea of the horizon. Then it emerged that the most educated blind people have such a knowledge of the architectural elements to allow them easy recognition even during exploration without directions (questionnaire 1); the same applies to those who have lost their sight in adolescence or adulthood and still retain visual memory

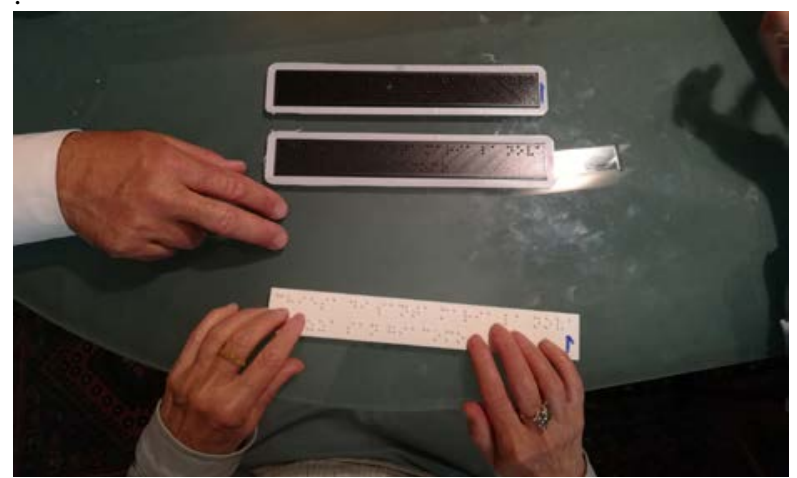

Figure 10. Test on different printed types of braille

Those who do not have a high level of education or visual memory, on the other hand, find tactile exploration more meaningful with explanations (questionnaire 2). Moreover, the visually impaired, with partial blindness or severe sight impairment interviewed, are able to distinguish just the forms and have a minimal idea of the architectures It has come out that the best printing scale evaluated by all as is 1:50 with a high print resolution, but in cases of very large monuments the 1:75 scale is acceptable; the 1: 100 scale model is too small to understand and too large a model could be too invasive in the exhibition space. Same consideration for Braille, the best writing is the number 1 (standard braille dimensions) with hemispherical conformation and the standard height, for each character equal to $1 \mathrm{~cm}$ (see Figure 10).

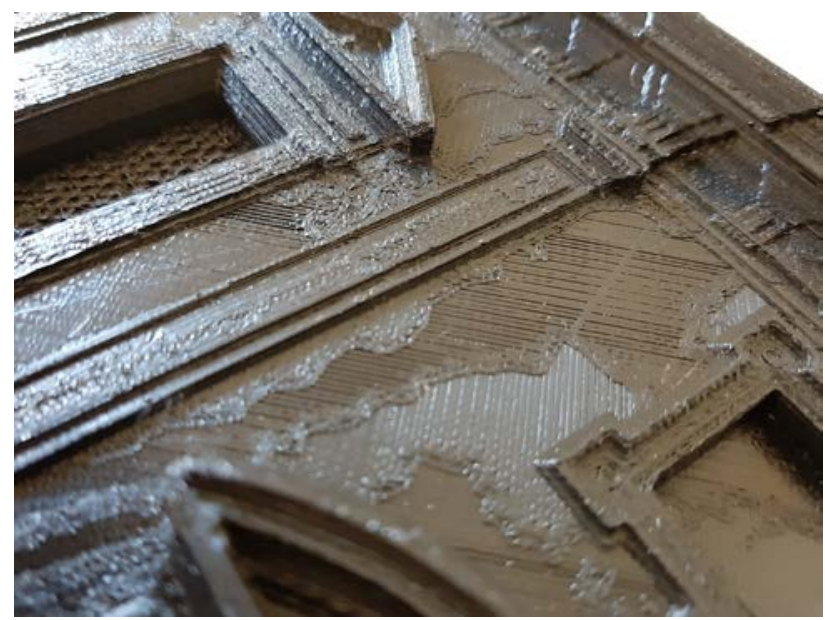

Figure 11. Detail of the deterioration of the facade

The surface finish of the masonry parts at a resolution of 6-10 $\mathrm{mm}$ in reading with a laser scanner and printing at the layer height of $0.10 \mathrm{~mm}$ is fine. As said by visually disabled people, increasing the roughness of the surfaces risks making other architectural parts less evident (pilasters and little projecting frames) (see Figure 11). Furthermore, it would be useful to have a two-tone high contrast reproduction for the visually impaired people models (yellow-black or white-black).

\section{CONCLUSIONS}

Laser scanner scanning, rapid prototyping and in particular 3D printing technologies offer great opportunities to bring back tactile experiences in our cultural heritage, extending accessibility in a "Design for All” perspective. It is possible to reproduce architectures starting from pieces broken down or in their entirety, placing these exposures in appropriate exhibition contexts. The ability to reproduce objects at a suitable scale and resolution, simplifies the tactile perception, making it easy for people with visual impairments to understand. In the context of the Design for All philosophy, all people benefit from these improvements and in particular those people who could not fully appreciate the original facades, such as blind or partially sighted visitors. In addition, the opportunity to extend accessibility to cultural assets to people with physical or visual disabilities can also have relapses and a positive economic, social, cultural and environmental impact. It would be interesting to equip the 3D models of sensors capable of giving indications on the architectural parts of the 3D model, so as to make tactile exploration even more accessible.

\section{FUNDING}

This research has been funded by Interreg I-ACCESS project "Implementing the Accessibility to Urban Historic Center's Use and Knowledge”, project code C1-1.1-41 - Axis 1.

\section{ACKNOWLEDGEMENTS}

We gratefully acknowledge Geom. Giovanni Abate and Geom. Davide Pellegrino of Leica Geosystem S.P.A. (Italy) for their precious support in TLS surveys.

\section{REFERENCES}

Amiralian, M. L. T. M., 1997. Compreendendo o cego. Casa do psicologo. Editora Casa do Psicólogo, Ano de publicação: 1997 $1^{\text {a }}$ Edição, ISBN: 85-7396-005-1

Amiralian, M.L.T.M., 2009. Deficienta visual: perspectivas na contemporaneidade. Vetor, Sao Paulo.

Balletti, C., Ballarin, M., Guerra, F., 2017. 3D printing: State of the art and future perspectives. Journal of Cultural Heritage, 26, 172-182.

Neumüller M., Reichinger A., Rist F., Kern C., 2014. 3D Printing for Cultural Heritage: Preservation, Accessibility, Research and Education. In: Ioannides M., Quak E. (eds) 3D Research Challenges in Cultural Heritage. Lecture Notes in Computer Science, vol 8355. Springer, Berlin, Heidelberg.

Dore, C., Murphy, M., 2012. Integration of HBIM and 3D GIS for Digital Heritage Modelling. In: Virtual Systems and Multimedia (VSMM), 2012 18th International Conference, Edimburg, Scotland: IEEE, 22-23.

Buehler, E., Comrie, N., Hofmann,M., McDonald, S., Hurst, A.,. 2016. Investigating the implications of $3 \mathrm{D}$ printing in special 
education. ACM Transactions on Accessible Computing 8, 3 (Mar. 2016), 1-28.

Hatwell, Y., Streri, A., Gentaz, E. (Eds.), 2003. Touching for knowing: cognitive psychology of haptic manual perception (Vol. 53). John Benjamins Publishing.

Heller, M. A., 1991. Haptic perception in blind people. The psychology of touch, 239-261.

Hollins, M., 1985. Styles of mental imagery in blind adults. Neuropsychologia, 23(4), 561-566.

Medline Plus: Blindness and vision loss, 2014, https://medlineplus.gov/ency/article/003040.htm.

Neumüller, M., Reichinger, A., Rist, F., \& Kern, C., 2014. 3D printing for cultural heritage: Preservation, accessibility, research and education. In $3 D$ Research Challenges in Cultural Heritage (pp. 119-134). Springer, Berlin, Heidelberg.

Peloso, D., 2005. Tecniche laser scanner per il rilievo dei beni culturali. Archeologia e calcolatori, (XVI), 199-224.

Reichinger, A., Fuhrmann, A., Maierhofer, S. and Purgathofer, W., 2016. Gesture-Based Interactive Audio Guide on Tactile Reliefs. ASSETS '16, 91-100.

Remondino, F., 2011. Heritage recording and 3D modeling with photogrammetry and 3D scanning. Remote Sensing, 3(6), 11041138.
Shi, L., 2015. Talkabel: A Labeling Method for 3D Printed Models. ASSETS '15, 361-362.

Shi, L., McLachlan, R., Zhao, Y. and Azenkot, S., 2016a. Magic Touch: Interacting with 3D Printed Graphics. ASSETS '16, 329330.

Shi, L., Zelzer, I., Feng, C. and Azenkot, S., 2016b. Tickers and Talker: An Accessible Labeling Toolkit for 3D Printed Models. CHI '16, 4896-4907.

Tinti, C., Adenzato, M., Tamietto, M., Cornoldi, C., 2006. Visual experience is not necessary for efficient survey spatial cognition: evidence from blindness. The Quarterly Journal of Experimental Psychology, 59(7), 1306-1328. 